\title{
Ausência de Correlação entre as Alterações Morfológicas e Bioquímicas na Microcirculação de Pacientes com Esclerose Sistêmica ${ }^{*}$
}

\section{Absence of Correlation between Morphological and Biochemical Alterations in the Microcirculation of Patients with Systemic Sclerosis}

\author{
Cristiane Kayser( ${ }^{(1)}$ e Luís Eduardo Coelho Andrade( ${ }^{(2)}$
}

\section{RESUMO}

Objetivo: A medida da lacticemia de polpa digital sob estímulo frio (LPD-EF) avalia um componente bioquímico da microcirculação, apresentando resultados anormais na esclerose sistêmica (ES). A capilaroscopia periungueal (CPU) é um método estabelecido para avaliação do componente morfológico no fenômeno de Raynaud secundário à ES. No presente estudo pretende-se a confrontação dos achados da capilaroscopia periungueal com os dados da LPD-EF em dedos individuais de pacientes com esclerose sistêmica. Métodos: Foram avaliados 14 pacientes com diagnóstico de ES e que apresentassem 3 dedos com grau discrepante de microangiopatia SD à CPU. Foi realizada dosagem da lacticemia de polpa digital nos três dedos selecionados em condições basais (LPD pré-EF) e 10 minutos após estímulo frio (LPD pós-EF). A variação percentual das medidas de LPD pós-EF em relação à LPD pré-EF foi denominada $\triangle$ LPD. Resultados: Houve acentuada variabilidade intraindividual (entre os diversos dedos) nos parâmetros do teste do LPD-EF. Não encontramos diferença estatisticamente significante nos valores da LPD pré-EF, pós-EF ou $\triangle \mathrm{LPD}$ ao compararmos os dedos com padrão capilaroscópico semelhante com os dedos díspares nos 14 pacientes com ES. Não houve correlação entre a intensidade dos achados morfológicos, registrados pela CPU, e as alterações funcionais, medidas pelo teste LPD-EF. Conclusões: A ausência de correlação entre as alterações microangiopáticas morfológicas verificadas pela CPU e as alterações funcionais de perfusão, avaliadas pelo teste da LPD-EF, sugere a possibilidade de que as últimas estejam relacionadas a lesões vasculares proximais, como arteríolas, artérias digitais e arco palmar.

Palavras-chave: esclerose sistêmica, fenômeno de Raynaud, capilaroscopia periungueal, microcirculação.

\begin{abstract}
Objective: The cold stimulus-fingertip lacticemy test (CS-FTL) evaluates a microcirculation biochemical component and presents abnormal results in systemic sclerosis (SSc). Nailfold capillaroscopy is a well established method for the assessment of morphological aspects of Raynaud's phenomenon associated with SSc. The present study sought to compare the nailfold capillaroscopy findings with the CS-FTL parameters in individual fingers of patients with systemic sclerosis. Methods: 14 SSc patients with 3 fingers having discrepant degree of microangiopathic SD pattern by nailfold capillaroscopy were studied. Fingertip lacticemy was determined in the three fingers selected before (pre-CS-FTL) and 10 minutes after cold stimulus (post-CS-FTL). The percentage difference between post- and pre-CS-FTL was designated $\triangle C S-F T L$. Results: There was great intraindividual variability (between the different digits) in values of the CS-FTL test. There was no statistically significant difference in pre-CS-FTL, post-CS-FTL and $\triangle C S-F T L$ values when comparing the fingers with similar capillaroscopic pattern and the discrepant finger in the 14 SSc patients. There was no correlation between the morphological findings registered by nailfold capillaroscopy and the functional alterations measured by the CS-FTL test. Conclusions: The absence of correlation between morphological microangiopathic alterations detected by nailfold capillaroscopy and the functional perfusion alterations, verified by the CS-FTL test, suggests a relationship between the latter and proximal vascular lesions, such as arterioles, digital and palmar arch arteries.
\end{abstract}

Keywords: systemic sclerosis, Raynaud's disease, nailfold capillaroscopy, microcirculation.

\footnotetext{
* Trabalho realizado na Disciplina de Reumatologia, Escola Paulista de Medicina, Universidade Federal de São Paulo (UNIFESP). Recebido em 22/09/03. Aprovado, após revisão, em 13/01/04. Este trabalho contou com o apoio da Fundação de Amparo à Pesquisa do Estado de São Paulo (FAPESP) e da Fundação de Apoio à Pesquisa da Sociedade Brasileira de Reumatologia.

1. Pós-graduanda em nível de Doutorado em Medicina. (UNIFESP).

2. Professor Adjunto - Livre Docente. (UNIFESP).
}

Endereço para correspondência: Dra.Cristiane Kayser. Av. Macuco, 518 - ap. 212, CEP 04523-001. São Paulo, SP, Brasil. 


\section{INTRODUÇÃO}

O fenômeno de Raynaud (FRy) é considerado a manifestação mais freqüente e precoce da esclerose sistêmica (ES), ocorrendo em até $95 \%$ dos pacientes ${ }^{(1)}$. Caracteriza-se por episódios reversíveis de vasoespasmo de extremidades associado a alterações de coloração típicas nas mãos e/ou pés, usualmente após contato com o frio ou estresse emocional. Enquanto no FRy primário o evento é benigno, havendo tipicamente ausência de dano tecidual ${ }^{(2,3)}$, no FRy secundário à ES alterações estruturais vasculares características somam-se ao vasoespasmo arterial podendo acarretar dano tecidual irreversível como reabsorção de polpa e falange distal, ulcerações, cicatrizes e gangrena de extremidades ${ }^{(4,5)}$. Lesões vasculares caracterizadas principalmente por proliferação miointimal e disfunção endotelial estão presentes nas pequenas artérias e na microcirculação já nos estágios iniciais da doença ${ }^{(5,6)}$.

A microangiopatia periférica da ES é bem documentada pela capilaroscopia periungueal (CPU), que consiste na visualização in vivo da rede microvascular na região periungueal. É um método já bem estabelecido para a avaliação do componente morfológico da microcirculação em pacientes com $\mathrm{ES}^{(7)}$. Os pacientes com FRy associado a doenças do espectro da esclerose sistêmica apresentam um quadro microangiopático específico, denominado padrão SD, caracterizado pela presença de dilatação (ectasia) e distorção capilar associado a áreas de completa desvascularização, além de diminuição difusa da quantidade de alças ${ }^{(8,9)}$ (Figuras 1A e 1B). Uma série de estudos demonstra seu valor para o diagnóstico da ES e síndromes correlatas, bem como para a detecção precoce de síndromes indiferenciadas auto-imunes que cursem com FRy ${ }^{(10-12)}$.

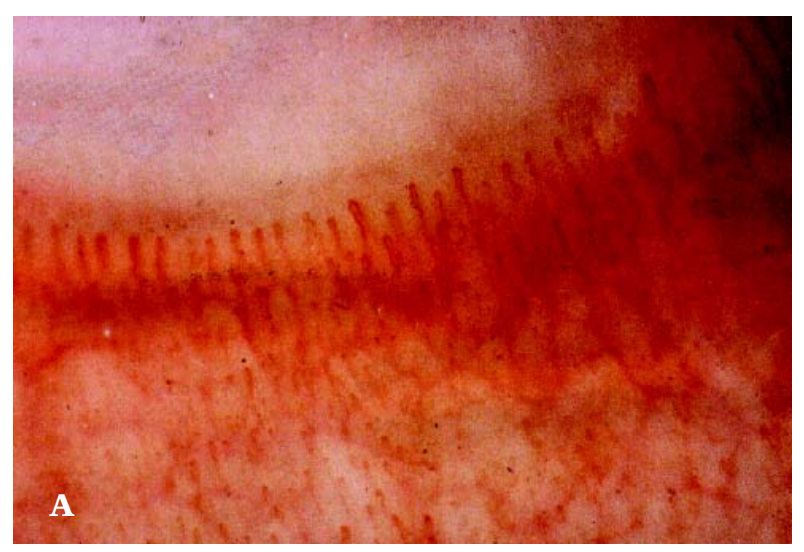

FigURA 1A - Capilaroscopia periungueal normal.

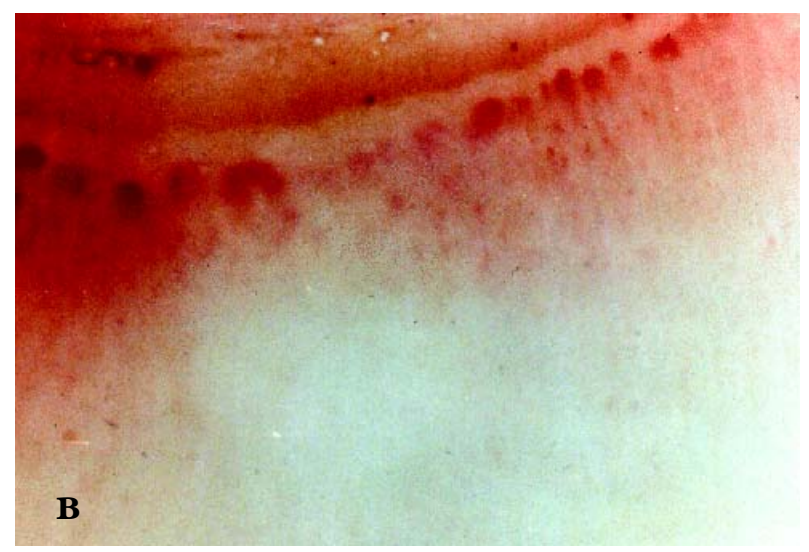

FiguRA 1B - Capilaroscopia periungueal com padrão SD. Observa-se presença de capilares ectasiados ao lado de áreas de desvascularização.

A técnica de dosagem da lacticemia em sangue de polpa digital (LPD), tradicionalmente utilizada para monitorização do treinamento aeróbico de atletas, foi originalmente adaptada pelo nosso grupo para avaliação quantitativa de um componente bioquímico da microcirculação em reumatologia ${ }^{(13,14)}$.

O aumento da lacticemia, quando excluídas outras causas que possam ocasionar a sua elevação, é um indicador de hipóxia tecidual. As células necessitam de um aporte contínuo de energia para manterem suas funções ${ }^{(15)}$. A via glicolítica é o principal sistema de liberação de energia da molécula de glicose. Normalmente, em condições de adequada perfusão e suprimento de oxigênio, a glicose se transforma em ácido pirúvico no citosol, entra então no ciclo dos ácidos tricarboxílicos onde ocorre a formação de dióxido de carbono e átomos de hidrogênio; estes são subseqüentemente oxidados (fosforilação oxidativa), ocorrendo liberação de grandes quantidades de energia para formar $\mathrm{ATP}^{(15,16)}$. Em certas ocasiões, como no exercício intenso $^{(17,18)}$ ou em situações de hipóxia tecidual ${ }^{(16,19)}$, o oxigênio deixa de ser disponível ou torna-se insuficiente, impedindo a ocorrência da fosforilação oxidativa; a maior parte do ácido pirúvico será então convertida em ácido láctico, acarretando o aumento da concentração sangüínea do mesmo ${ }^{(16)}$. Assim, as principais causas de aumento da produção do lactato são: aumento intenso e rápido na taxa metabólica, como no exercício intenso ${ }^{(17,18)}$, estados de choque, sépsis, insuficiência cardíaca ou edema agudo de pulmão (situações de hipóxia tecidual) ${ }^{(16,19)}$ e mais raramente na administração de certas drogas, como as catecolaminas, ou em erros inatos do metabolismo, como a deficiência da enzima piruvato desidrogenase ${ }^{(16)}$. 
$\mathrm{O}$ teste denominado lacticemia de polpa digital associada a estímulo frio (LPD-EF) consiste na dosagem da LPD em condições basais e 10 minutos após um estímulo frio (EF) (imersão das mãos em água a $10^{\circ} \mathrm{C}$ por 1 minuto) ${ }^{(13)}$. $\mathrm{O}$ teste LPD-EF foi recentemente padronizado para a população geral brasileira através do estudo da LPD em diferentes intervalos de tempo após o EF e foram feitas análises de possíveis variações em relação ao gênero e idade. Na ES a LPD basal foi superior em comparação aos normais $(2.68 \pm 1.33 \mathrm{mg} / \mathrm{dL}$ versus 1.98 $\pm 0.59 \mathrm{mg} / \mathrm{dL}, \mathrm{p}<0.001)$. Além disso, a LPD pós-EF apresentou queda significativa na maioria dos indivíduos normais e tendência à elevação na maioria dos pacientes com ES, mostrando um comportamento dinâmico oposto da LPD frente a um estímulo frio entre os dois grupos. A variação percentual das medidas de LPD pós-EF em relação à LPD pré$\mathrm{EF}$, denominada de $\triangle \mathrm{LPD}$, foi utilizada para o estabelecimento do ponto de corte ideal ( $\Delta$ LPD em -10\%) para a discriminação entre pacientes com ES e controles normais, sendo que valores de $\triangle \mathrm{LPD} \geq-10 \%$ foram considerados alterados ${ }^{(14)}$. O teste da LPD-EF mostrou-se assim, acurado e potencialmente útil para diagnóstico da ES.

Uma observação intrigante nos pacientes com FRy secundário à ES é a heterogeneidade que se observa entre os vários dedos de um mesmo indivíduo. Alguns dedos apresentam episódios de FRy mais freqüentes e intensos que outros. A incidência dos fenômenos tróficos também é extremamente variável. Além disso, a observação capilaroscópica via de regra flagra diferenças morfológicas acentuadas entre dedos vizinhos. Tal heterogeneidade é ainda mais intrigante quando se considera que as variáveis genéticas, ambientais, terapêuticas e de evolução de doença são fixas no indivíduo.

Por isso, achamos válido confrontar dedos morfologicamente heterogêneos à CPU com o método da LPD-EF a fim de se verificar se as alterações morfológicas da microcirculação estão associadas aos achados bioquímicos da LPDEF. Esta informação teria o potencial de avaliar as possíveis conseqüências funcionais e bioquímicas da microangiopatia SD. No presente estudo comparamos as alterações de perfusão sanguínea mediante dosagem da lacticemia de polpa digital antes e após estímulo frio (LPD-EF), em pacientes com ES que apresentassem dedos com diferentes graus de microangiopatia SD definida pela CPU.

\section{MATERIAIS E MÉTODOS}

Foram avaliados consecutivamente pacientes oriundos do Ambulatório de Doenças do Espectro da Esclerodermia do
HSP-UNIFESP com diagnóstico de ES segundo os critérios de classificação da $\mathrm{ACR}^{(20)}$, e que tinham padrão $\mathrm{SD}$ à $\mathrm{CPU}$.

Foram excluídos os seguintes pacientes: 1) tabagistas; 2) pacientes em uso de antiagregantes plaquetários, anticoagulantes e anticoncepcional oral; 3) portadores de arteriopatia arteriosclerótica periférica, diabéticos, dislipidêmicos, tireoidopatas e pneumopatas; 4) indivíduos cuja ocupação os expusesse sistematicamente ao frio ou a instrumentos vibratórios. Qualquer medicação vasodilatadora utilizada pelos pacientes foi suspensa três dias antes da realização dos testes.

Todos os pacientes deveriam ter maioridade e ter assinado o termo ético de consentimento informado aprovado pela Comissão de Ética da UNIFESP.

\section{Seleção de dedos com graus discrepantes de microan-} giopatia SD: Inicialmente, foi feita avaliação capilaroscópica dos pacientes, através da qual foram selecionados consecutivamente para o estudo 14 indivíduos que apresentaram padrão SD à CPU. Três dedos da mesma mão deveriam ter as seguintes características: dois dedos com padrão capilaroscópico semelhante entre si (dedos A e B), e um terceiro com acentuada disparidade em relação aos dois primeiros (dedo C). A classificação de semelhança ou disparidade capilaroscópica interdigital foi baseada na avaliação de três parâmetros, a saber:

1) ectasia capilar: alças alargadas em todos os seus três ramos - aferente, transição e eferente, correspondendo a cerca de quatro vezes maior que o normal ${ }^{(21)}$;

2) megacapilar: correspondem a alças extremamente alargadas com calibre dez vezes maior que as alças adjacentes normais ${ }^{(21)}$;

3) deleção: para quantificar o grau de deleção ou área avascular, seguimos a escala de Lee et al..$^{(9)}$ que define como deleção a ausência de dois ou mais capilares sucessivos, permitindo quantificar este parâmetro numa escala de $0 \mathrm{a}$ 3 conforme a extensão das lesões: 0 - nenhuma área de deleção; 1 - uma ou duas áreas de deleção descontínuas; 2 - mais de duas áreas de deleção descontínuas; 3 - áreas extensas e confluentes de desvascularização.

Foram considerados semelhantes os dedos com o mesmo grau de deleção e cuja diferença no número de capilares ectasiados fosse menor que 5 e de megacapilares menor que 1. Inversamente, foram considerados díspares os dedos com um dos seguintes parâmetros: grau de deleção diferente ou diferença no número de capilares ectasiados $\geq 5$ ou de megacapilares $\geq 1$. 
Teste de dosagem da lacticemia de polpa digital associada a estímulo frio (LPD-EF): Foi dividido em 4 etapas: Etapa 1) repouso por 30 minutos em ambiente à temperatura de $24 \pm 1^{\circ} \mathrm{C}$; Etapa 2) dosagem da lacticemia de polpa digital (LPD) realizada nos três dedos selecionados através de lanceta acoplada ao aparelho Softclix (Boehringer Mannheim, Alemanha); faz-se a punção em polpa digital, colhendo-se a primeira gota de sangue (sem efetuar ordenha local) diretamente sobre a região demarcada na tira para dosagem do lactato. A leitura é realizada imediatamente, inserindo-se a fita no espectrofotômetro de reflexão Accusport (Boehringer Mannheim, Alemanha); Etapa 3) estímulo frio através de imersão das mãos em água à $10^{\circ} \mathrm{C}$ por 60 segundos (UNITEMP 116, Fanem, Brasil), realizada logo após a leitura da LPD; Etapa 4) monitoração de LPD, realizada no décimo minuto nos três dedos selecionados de forma semelhante ao item 2 .

\section{DESENHO METODOLÓGICO E ANÁLISE ESTATÍSTICA}

A fim de melhor evidenciar as alterações dinâmicas da LPD em função do EF, utilizou-se também a variação percentual das medidas de LPD pós-EF em relação à LPD pré-EF, denominada de $\triangle \mathrm{LPD}$, onde:

$$
\Delta \mathrm{LPD}=\frac{\text { lacticemia pós-EF - lacticemia pré-EF } \times 100}{\text { lacticemia pré-EF }}
$$

A comparação das variáveis quantitativas foi feita pelo teste t-pareado. O coeficiente de correlação de Spearman foi utilizado para se avaliar a correlação entre as alterações à CPU e o $\triangle \mathrm{LPD}$. Para todas as análises foi considerado o valor de significância menor que 0,05 .

\section{RESULTADOS}

Foram avaliados 14 pacientes com ES com média de idade de 48,6 anos (29-73 anos); 12 pacientes (86\%) eram do sexo feminino e dois (14\%) do sexo masculino. Seis pacientes apresentaram diagnóstico de ES forma difusa e oito pacientes apresentaram a forma limitada.

Conforme os dados da Tabela 1, não encontramos diferença estatisticamente significante nos valores de $\Delta$ LPD ao compararmos os dedos com padrão capilaroscópico semelhante entre si (dedo A versus dedo B; $-2,45 \pm 21,33 \%$ versus $+2,08 \pm 18,95 \%$ ), ou ao compararmos os dedos semelhantes com os dedos díspares (dedo A versus dedo $\mathrm{C} ;-2,45 \pm 21,33 \%$ versus $+3,22 \pm 31,29 \%$ ) (dedo B versus dedo $C ;+2,08 \pm 18,95 \%$ versus $+3,22 \pm 31,29 \%)$ nos 14 pacientes estudados.
Numa análise da Tabela 1, observa-se que alguns pacientes apresentaram o $\triangle$ LPD do dedo díspar com valores bastante discrepantes em relação aos dois dedos semelhantes entre si. Entretanto, vários outros pacientes apresentaram $\Delta$ LPD semelhante nos três dedos, corroborando a ausência de diferença sistemática no teste LPD-EF entre dedos com diferentes graus de microangiopatia à capilaroscopia periungueal.

TABELA 1

VARIAÇÃO PERCENTUAL DA LPD PÓS-EF EM RELAÇÃO A LPD PRÉ-EF ( $\triangle \mathrm{LPD})$, NOS DEDOS SEMELHANTES (A E B) E NO DEDO DíSPAR (C) À CAPILAROSCOPIA PERIUNGUEAL NOS PACIENTES COM ES.

\begin{tabular}{|c|c|c|c|}
\hline & \multicolumn{2}{|c|}{ Dedos semelhantes } & Dedo díspar \\
\hline Paciente & A & $B$ & $C$ \\
\hline 1 & $-40,5 \%$ & $-44,2 \%$ & $-13,3 \%$ \\
\hline 2 & $-22,9 \%$ & $+16,3 \%$ & $-42,3 \%$ \\
\hline 3 & $-28,2 \%$ & $-14,3 \%$ & $-57,1 \%$ \\
\hline 4 & $+8,6 \%$ & $+23,4 \%$ & $+7,3 \%$ \\
\hline 5 & $+9,1 \%$ & $+19,2 \%$ & $+4,3 \%$ \\
\hline 6 & $+3,3 \%$ & $-3,0 \%$ & $0,0 \%$ \\
\hline 7 & $+29,2 \%$ & $+6,2 \%$ & $+34,8 \%$ \\
\hline 8 & $-14,3 \%$ & $-25,0 \%$ & $-18,8 \%$ \\
\hline 9 & $+15,8 \%$ & $+4,5 \%$ & $+37,5 \%$ \\
\hline 10 & $+7,7 \%$ & $+21,4 \%$ & $+46,7 \%$ \\
\hline 11 & $0,0 \%$ & $0,0 \%$ & $-16,7 \%$ \\
\hline 12 & $+8,3 \%$ & $+6,5 \%$ & $+43,5 \%$ \\
\hline 13 & $-31,6 \%$ & $+5,0 \%$ & $-7,1 \%$ \\
\hline 14 & $+21,1 \%$ & $+13,0 \%$ & $+26,3 \%$ \\
\hline $\begin{array}{l}\text { Média } \pm \\
\text { desvio padrão }\end{array}$ & $-2,45 \pm 21,33$ & $+2,08 \pm 18,95$ & $+3,22 \pm 31,29$ \\
\hline
\end{tabular}

A média dos valores da LPD pré-EF nos dedos semelhantes foi de $2.77 \pm 1.16 \mathrm{mmol} / \mathrm{L}$ e nos dedos díspares de $2.67 \pm 1.66 \mathrm{mmol} / \mathrm{L}$. Já a média dos valores da LPD pós-EF nos dedos semelhantes foi de $2.74 \pm 1.27 \mathrm{mmol} / \mathrm{L}$ e nos dedos díspares de $2.74 \pm 1.87 \mathrm{mmol} / \mathrm{L}$. Não encontramos diferença estatisticamente significante nos valores da LPD pré-EF, LPD pós-EF ou $\triangle \mathrm{LPD}$ ao compararmos todos os 28 dedos semelhantes (dedo A e B) com os 14 dedos díspares à CPU (dedo C) (Tabela 2). 
TABELA 2

VALORES DE LPD PRÉ-EF, LPD PÓS-EF E $\triangle L P D$ (MÉDIA \pm DESVIO PADRÃO), CONFORME O GRAU DE SEMELHANÇA OU DISPARIDADE À CAPILAROSCOPIA PERIUNGUEAL (CPU).

\begin{tabular}{lccc}
\hline & \multicolumn{4}{c}{ Grau de microangiopatia SD à CPU } \\
\hline & $\begin{array}{c}\text { Dedos semelhantes } \\
(\mathrm{n}=28)\end{array}$ & $\begin{array}{c}\text { Dedo díspar } \\
(\mathrm{n}=14)\end{array}$ & $\mathrm{p}=0.65$ \\
\hline LPD pré-EF $(\mathrm{mmol} / \mathrm{L})$ & $2.77 \pm 1.16$ & $2.67 \pm 1.66$ & $\mathrm{p}=1.00$ \\
LPD pós-EF $(\mathrm{mmol} / \mathrm{L})$ & $2.74 \pm 1.27$ & $2.74 \pm 1.87$ & $\mathrm{p}=0.47$ \\
\hline LPD $(\%)$ & $-0.19 \pm 19.94$ & $3.22 \pm 31.29$ & \\
\hline
\end{tabular}

A possibilidade de correlação entre a intensidade da microangiopatia SD e as alterações de perfusão tecidual, representadas pela lacticemia de polpa digital, foi avaliada mediante análise de dispersão de Spearman entre parâmetros capilaroscópicos e variáveis do teste LPD-EF. Como se verifica na Figura 2, não houve correlação entre os graus de ectasia e deleção à capilaroscopia periungueal e os valores de LPD pré-EF e $\triangle \mathrm{LPD}$.
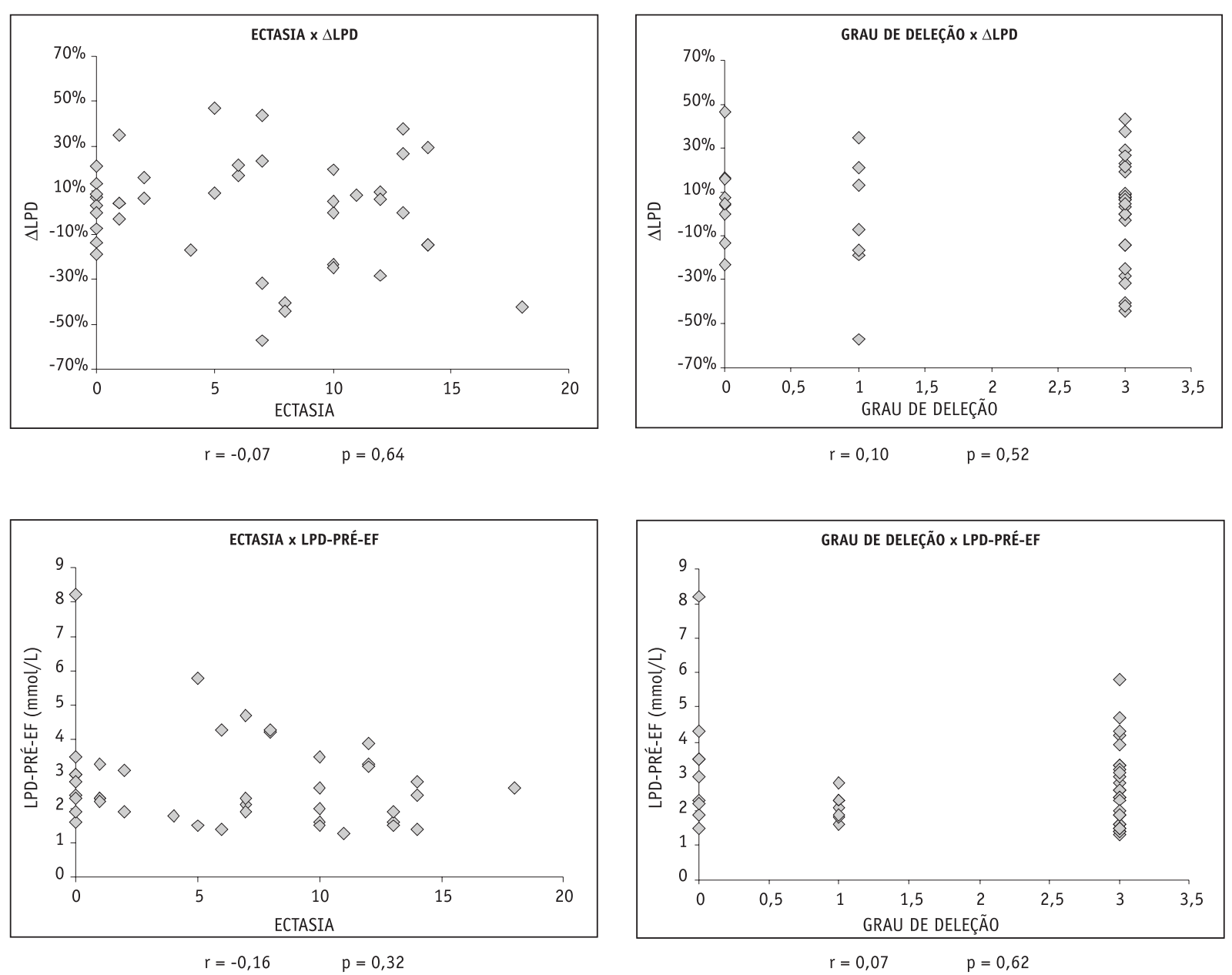

Figura 2 - Gráficos correlacionando os valores de $\Delta$ LPD (\%) e LPD pré-EF (mmol/L) com a quantidade de capilares ectasiados e o grau de deleção nos 42 dedos avaliados. 


\section{DISCUSSÃO}

Focalizamos este presente estudo em dois métodos que contemplam dois aspectos diferentes da microcirculação: a capilaroscopia periungueal, que avalia de forma rápida e nãoinvasiva o aspecto anatômico, e a LPD-EF. O teste da LPD-EF, recentemente descrito por nós, é um método quantitativo que fornece uma dimensão funcional da circulação distal de pacientes com ES, ou seja, reflete o aporte de oxigênio dos vasos ao tecido e sua utilização pelas células.

Os valores da LPD pré-EF, LPD pós-EF e $\triangle \mathrm{LPD}$ encontrados no presente estudo, estão em conformidade com os valores descritos anteriormente ${ }^{(13)}$. Os pacientes com ES apresentam LPD basal mais elevada com relação aos controles normais, o que parece ser reflexo da diminuição do fluxo sangüíneo e da hipóxia crônica de extremidades descrita nesta doença.

Além disso, ao se analisar o $\triangle$ LPD nos 42 dedos estudados, observamos que $69 \%$ dos valores de $\triangle \mathrm{LPD}$ apresentaramse alterados após o estímulo frio $(\Delta \mathrm{LPD} \geq-10 \%)$, corroborando a presença de alterações na dinâmica do fluxo sangüíneo capilar na ES. Devemos ressaltar, entretanto, o comportamento heterogêneo da LPD frente a um estímulo frio nos pacientes estudados: um número considerável destes $(31 \%)$ apresentou queda da LPD após o EF $(\Delta \mathrm{LPD}<-10 \%)$, comportamento este compatível com a normalidade. Verificamos também um desvio padrão acentuado nos valores de $\triangle$ LPD obtidos nos 42 dedos estudados. O fato de não termos tido um comportamento homogêneo da LPD após estímulo frio nos pacientes esclerodérmicos está em conformidade com resultados descritos na literatura que utilizaram outros métodos de avaliação do FRy. É bem descrita uma acentuada heterogeneidade interindividual no comportamento da microcirculação frente a um estímulo frio $^{(22-24)}$. Nossos achados salientam também a acentuada variabilidade intraindividual, ou seja, entre dedos distintos de um mesmo paciente.

Conforme Cleophas et al. ${ }^{(25)}$ fatores como baixa temperatura ambiente, duração da aclimatização e tabagismo influenciam a resposta ao estímulo frio. Estes fatores podem contribuir para a baixa reprodutibilidade e grande variabilidade dos resultados entre diferentes estudos. Em nosso trabalho, tentamos minimizar a influência de fatores extrínsecos, através de um protocolo homogêneo aplicado a todos os pacientes e constituído por aclimatização por no mínimo 30 minutos em ambiente tranquiilo e temperatura rigorosamente estabelecida em $24^{\circ} \mathrm{C}$. Também excluímos todos os tabagistas do estudo.
O aumento da LPD após EF observado na maioria dos pacientes com ES pode ser decorrente de alterações na dinâmica do fluxo sangüíneo encontrada nesses indivíduos. Ao se fazer um estímulo frio, os pacientes esclerodérmicos apresentam uma resposta sabidamente exacerbada à isquemia aguda, com um aumento abrupto do tônus vascular, diminuição acentuada do fluxo sangüíneo e até um possível fechamento das arteríolas digitais ${ }^{(26)}$. A diminuição do fluxo sangüíneo periférico provocada pelo estímulo frio associada à lentificação do fluxo sangüíneo próprio da ES, favoreceria um regime de hipóxia e intensa troca de metabólitos entre o sangue capilar e os tecidos, que se refletiria num aumento da LPD. Vale ressaltar novamente que os valores basais da LPD já são mais elevados nos pacientes com ES, corroborando os achados que demonstram alterações morfológicas conspícuas, desvascularização e diminuição do fluxo sangüíneo na pequena e microcirculação desses indivíduos ${ }^{(5,6)}$.

A pergunta central do presente estudo refere-se à relação entre as alterações morfológicas da microcirculação observadas à CPU e as alterações de perfusão tecidual registradas pelo teste da LPD-EF. Poderíamos esperar que quanto mais intensas fossem as alterações capilaroscópicas, mais anormais os valores de LPD. Tal achado falaria a favor de um papel fisiopatológico direto da microangiopatia capilar da esclerose sistêmica. Entretanto, nossos achados não subsidiam esta linha de raciocínio. Pelo contrário, encontramos total desconexão entre os achados morfológicos da CPU e as alterações de perfusão registradas pelo teste LPD-EF. Os valores da LPD pré e pós-EF e de $\triangle$ LPD se comportaram de modo semelhante ao se comparar dedos com intensa microangiopatia à CPU com dedos com discretas ou até mesmo nenhuma alteração capilaroscópica.

A CPU e a medida da lacticemia de polpa digital associada a um estímulo frio são métodos distintos que analisam componentes diferentes da microcirculação. As alterações à CPU representam alterações morfológicas estruturais da microcirculação especificamente no nível dos capilares, e persistem por semanas a meses após exames consecutivos. Portanto, essas alterações caracterizam lesões estruturais relativamente insensíveis a estímulos vasomotores ${ }^{(21)}$. Já a medida da LPD, antes e após um estímulo frio, avalia um componente funcional bioquímico da perfusão tecidual altamente responsivo a fenômenos vasoespásticos, podendo ser considerado um método mais dinâmico se comparado com a CPU. Por analisar as consequiências bioquímicas da respiração anaeróbica celular, o teste LPD-EF traduz de forma pragmática a eficiência da perfusão tecidual. 
$\mathrm{O}$ achado do presente estudo, de que não houve correlação entre a intensidade das alterações morfológicas (CPU) e funcionais (perfusão tecidual medida pelo teste LPD-EF), sugere que as últimas talvez se devam a anormalidades do leito vascular a montante da rede capilar. A literatura tem documentado lesões relevantes como estenose e oclusão de artérias digitais e arco palmar superficial em pacientes com $\mathrm{ES}^{(27,28)}$. Assim, tendo em mãos os presentes resultados, cogitamos sobre a

\section{REFERÊNCIAS}

1. Seibold JR, Smith EA, Leroy EC, Steen VD: Systemic sclerosis. Em Klippel, JH, Dieppe, PA (editores): Rheumatology. $1^{\text {a }}$ edição, Londres, Mosby, 1994

2. Allen EV, Brown GE: Raynaud's disease: a critical review of minimal requisites for diagnosis. Am J Med Sci 183: 187-200, 1932.

3. LeRoy EC, Medsger Jr TA: Raynaud's phenomenon: a proposal for classification. Clin Exp Rheumatol 10: 485-8, 1992.

4. Campbell PM, LeRoy EC: Pathogenesis of systemic sclerosis: a vascular hypothesis. Semin Arthritis Rheum 4: 351-68, 1975.

5. Prescott RJ, Freemont AJ, Jones CJP, Hoyland J, Fielding P: Sequential dermal microvascular and perivascular changes in the development of scleroderma. J Pathol 166: 255-63, 1992.

6. Burch GE, Harb JM, Sun CS: Fine structure of digital vascular lesions in Raynaud's phenomenon and disease. Angiology 21: 361-76, 1981.

7. Andrade LEC, Gabriel Jr A, Assad RL, Ferrari JAL, Atra E: Panoramic nailfold cappilaroscopy: a new reading method and normal range. Semin Arthritis Rheum 20: 21-31, 1990.

8. Maricq HR, LeRoy EC, D’Angelo WA, et al: Diagnostic potential of in vivo capillary microscopy in scleroderma and related disorders. Arthritis Rheum 23: 183-9, 1980.

9. Lee P, Leung F, Alderdice C, Armstrong SK: Nailfold capillary microscopy in the connective tissue diseases: a semiquantitative assessment. J Rheumatology 10: 930-8, 1983.

10. Maricq HR, Harper FE, Khan MM: Microvascular abnormalities as possible predictors of disease subsets in Raynaud's phenomenon and early connective tissue disease. Clin Exp Rheumatol 1: 195-205, 1982.

11. Lovy M, MacCarter D, Steigerwald JC: Relationship between nailfold capillary abnormalities and organ involvement in systemic sclerosis. Arthritis Rheum 28: 496-501, 1985.

12. Pucinelli MLC, Atra E, Sato EI, Andrade LEC: Nailfold cappilaroscopy in systemic sclerosis: correlations with involvement of lung and esophagus. Rev Bras Reumatol 35: 136-42, 1995.

13. Pucinelli MLC, Fontenelle SMA, Andrade LEC: Determination of fingertip lacticemy before and after cold stimulus in patients with primary Raynaud's phenomenon and systemic sclerosis. J Rheumatol 29: 1401-3, 2002.

14. Kayser C: Padronização do teste de lacticemia de polpa digital antes e após estímulo frio na população geral e comparação com pacientes possibilidade de que as alterações bioquímicas apontadas pelo teste da LPD-EF na ES estejam associadas a irregularidades de artérias digitais e do arco palmar ou de arteríolas distais a essas, e de que as alterações capilaroscópicas estejam desvinculadas de ambas. Para elucidar estes pontos, pretendemos em um próximo estudo comparar os achados atuais, capilaroscópicos e de LPD-EF, com a arteriografia do arco palmar e de artérias digitais nos pacientes com ES.

com fenômeno de Raynaud secundário à esclerose sistêmica. Submetido à publicação.

15. Guyton AC: Metabolismo dos carboidratos e formação de trifosfato de adenosina. Em: Guyton AC: Tratado de Fisiologia Médica. 9a edição, Rio de Janeiro, Guanabara Koogan, 1997.

16. Toffaletti JG: Blood lactate: Biochemistry, laboratory methods, and clinical interpretation. Crit Rev Clin Lab Sciences 28: 253-68, 1991.

17. Karlsson J: Muscle exercise, energy metabolism and blood lactate. Adv Cardiol 35: 35-46, 1986.

18. Wasserman K, Beaver WL, Whipp BJ: Mechanisms and patterns of blood lactate increase during exercise in man. Med Sci Sports Exerc 18: 344-52, 1986.

19. Gutierrez G, Wulf ME: Lactic acidosis in sepsis: a commentary. Intensive Care Med 22: 6-16, 1996.

20. Masi AT, Rodnan GP, Medsger Jr TA, et al: Preliminary criteria for the classification of systemic sclerosis (scleroderma). Arthritis Rheum 23: 581-90, 1980.

21. Maricq HR: Widefield capillary microscopy. Technique and rating scale for abnormalities seen in scleroderma and related disorders. Arthritis Rheum 24: 1159-65, 1981.

22. Herrick AL, Clark S: Quantifying digital vascular disease in patients with primary Raynaud's phenomenon and systemic sclerosis. Ann Rheum Dis 57: 70-8, 1998.

23. Bartelink ML, Wollersheim H, Leesmans E, De Boo Th, Thien Th: A standardized finger cooling test for Raynaud's phenomenon: diagnostic value and sex differences. Eur Heart J 14: 614-22, 1993.

24. Maricq HR, Weinrich MC, Valter I, Palesch YY, MaricqJG: Digital vascular responses to cooling in subjects with cold sensitivity, primary Raynaud's phenomenon, or scleroderma spectrum disorders. J Rheumatol 23: 2068-78, 1996.

25. Cleophas TJM, Fennis JFM, Laar A: Finger temperature after a fingercooling test: influence of air temperature and smoking. J Appl Physiol 52: 1167-71, 1982.

26. Kurki TS, Piirainen HI, Kurki PT: Non-invasive monitoring of finger arterial pressure in patients with Raynaud's phenomenon: effects of exposure to cold. Br J Anaesth 65: 558-63, 1990.

27. Janevski B: Arteries of the hand in patients with scleroderma. Diagn Imaging Clin Med 55: 262-5, 1986.

28. Stucker M, Quinna S, Memmel U, et al: Macroangiopathy of the upper extremities in progressive systemic sclerosis. Eur J Med Res 5: 295-302, 2000 